\title{
Heterogeneous nuclear ribonucleoprotein $K$ is overexpressed and contributes to radioresistance irrespective of HPV status in head and neck squamous cell carcinoma
}

\author{
JUSTUS KÄHLER ${ }^{1,2}$, SUSANNE HAFNER ${ }^{3}$, TANJA POPP ${ }^{1}$, CORNELIUS HERMANN ${ }^{1}$, \\ ALEXIS RUMP $^{1}$, MATTHIAS PORT $^{1}$, KONRAD STEINESTEL $^{4}$ and STEFAN EDER ${ }^{1,2}$ \\ ${ }^{1}$ Bundeswehr Institute of Radiobiology, D-80937 Munich; ${ }^{2}$ Institute and Outpatient Clinic for Occupational, \\ Social and Environmental Medicine, Inner City Clinic, University Hospital of Munich (LMU), D-80336 Munich; \\ ${ }^{3}$ Institute of Pharmacology of Natural Products and Clinical Pharmacology, University of Ulm; \\ ${ }^{4}$ Institute of Pathology and Molecular Pathology, Bundeswehrkrankenhaus Ulm, D-89081 Ulm, Germany
}

Received May 7, 2020; Accepted August 17, 2020

DOI: $10.3892 / \mathrm{ijmm} .2020 .4718$

\begin{abstract}
Radiotherapy is a major treatment option for head and neck squamous cell carcinoma (HNSCC). However, the success of radiotherapy is limited by tumor cell resistance to ionizing radiation (IR). Clinical studies have demonstrated an overall improved prognosis and higher susceptibility to radiotherapy of high-risk human papillomavirus (HPV)-associated HNSCC compared with classic HNSCC, as well as worse overall survival for male HNSCC patients. Overexpression of heterogeneous nuclear ribonucleoprotein (hnRNP) $\mathrm{K}$ has been associated with resistance to radiotherapy in melanoma and colorectal carcinoma. The aim of the present study was to analyze the impact of hnRNP K expression on the aggressiveness and radioresistance of HNSCC with respect to patient sex and HPV status. Immunohistochemical staining of HNSCC tissue specimens revealed elevated hnRNP K levels compared with those in the non-neoplastic epithelium. Cytoplasmic hnRNP K accumulation was associated with advanced tumor stage and male sex. Exposure of HNSCC cells to IR was followed by rapid upregulation of hnRNP K at the protein level, along with re-localization from the tumor cell nucleus to the cytoplasm. siRNA-based knockdown of hnRNP K induced apoptosis and abolished tumor formation
\end{abstract}

Correspondence to: Dr Stefan Eder, Bundeswehr Institute of Radiobiology, 11 Neuherbergstrasse, D-80937 Munich, Germany

E-mail: stefanfriedricheder@bundeswehr.org

Abbreviations: CAM, chorioallantoic membrane; FFPE, formalin-fixed and paraffin-embedded; IF, immunofluorescence; IHC, immunohistochemistry; IR, ionizing radiation; hnRNP K, heterogenous nuclear ribonucleoprotein K; HNSCC, head and neck squamous cell carcinoma; HPV, human papillomavirus

Key words: head and neck squamous cell carcinoma, heterogenous nuclear ribonucleoprotein $\mathrm{K}$, radiation, tumorigenesis, sex after xenotransplantation of HNSCC cells onto the chick egg chorioallantoic membrane (CAM). The observed effects were independent of the respective HPV status of the cell lines. These results indicated a tumorigenic and anti-apoptotic role of hnRNP K in HNSCC, which appeared to be enhanced in male patients and contributed to the radioresistance of these tumors. However, the radioprotective effects of hnRNP K were found to be independent of the tumor's HPV status.

\section{Introduction}

Head and neck squamous cell carcinoma (HNSCC), including tumors arising in the nasopharynx, oropharynx and the oral cavity, are frequent malignancies with a worldwide incidence of $>650,000$ cases and 330,000 deaths per year $(1,2)$. HNSCC belongs to a family of tumors for which a strong association with combined alcohol and tobacco use has been established $(1,3,4)$. In contrast to the overall declining incidence rates, numerous epidemiological studies recently highlighted a marked increase of human papillomavirus (HPV)-associated HNSCC in various western countries, emerging as a growing public health concern (5-7). Interestingly, HPV-associated $\left(\mathrm{HPV}^{+}\right) \mathrm{HNSCC}$ appears to be more sensitive to radiotherapy, which contributes in part to the observed better prognosis $(4,8)$. Currently, the viral oncoproteins E6 and E7 are considered to be responsible for impaired DNA damage response upon exposure to ionizing radiation (IR) in $\mathrm{HPV}^{+} \mathrm{HNSCC}$ due to degradation of p53 and the inactivation of retinoblastoma family members, but the precise mechanism underlying the enhanced radiosensitivity of HPV-associated tumors remains elusive $(9,10)$.

Numerous studies have highlighted the impact of heterogeneous nuclear ribonucleoprotein (hnRNP) K as a key player in tumorigenesis $(11,12)$. This multifunctional protein acts as a highly integrated docking platform for various signaling pathways and regulates the expression of target genes, as well as the translation of mRNA transcripts associated with cancer initiation and progression $(12,13)$. High hnRNP K expression was repeatedly reported in various cancer tissues, 
including HNSCC, and linked to advanced tumor stage as well as enhanced invasiveness, increased propensity for metastasis and poor clinical outcome (14-19). Accordingly, it was reported that hnRNP K knockdown reduces the tumorigenic characteristics of multiple cancer cells (18).

Furthermore, hnRNP K represents a crucial co-factor for the p53-mediated DNA damage repair (DDR) upon exposure to IR. DNA damage finally results in the upregulation of transcripts for p53 target genes in a p53/hnRNP K-codependent manner (20). As a typical consequence, IR-induced arrest of cell cycle progression at the $\mathrm{G} 1$ or $\mathrm{G} 2$ phase is mediated by the

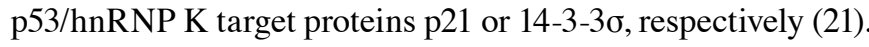

Finally, hnRNP K has been shown to be indispensable for an effective cellular IR-induced DDR in various cell models, including HNSCC $(22,23)$.

Interestingly, a recent proteomic study in HNSCC cells revealed increased cellular hnRNP K levels in response to IR, whereas hnRNP K knockdown impaired HNSCC cell migration, which is a prerequisite for metastasis (24).

The aim of the present study was to analyze whether hnRNP K expression contributes to the differences in radioresistance between $\mathrm{HPV}^{+}$and $\mathrm{HPV}^{-} \mathrm{HNSCC}$. It was hypothesized that the known responsiveness of HPV-dependent HNSCC to radiotherapy may be associated with higher expression of hnRNP K compared with HPV-independent HNSCC. Therefore, the functional consequences of hnRNP K in the tumorigenic characteristics of HNSCC cells were investigated applying immunohistochemistry (IHC), knockdown analyses and a chick egg chorioallantoic membrane (CAM) assay as a preclinical in vivo tumor model. Furthermore, as the prognosis of patients with HNSCC is associated with sex, potential differences in hnRNP K expression between HNSCC samples from female and male patients were investigated. The results of the present study may facilitate further stratification of HNSCC patients beyond HPV status with regard to dosage of radiotherapy and/or elucidate the role of hnRNP K and associated pathways as possible therapeutic targets in HNSCC.

\section{Materials and methods}

Tissue samples. Two slides each from two head and neck cancer tissue microarrays (TMA) were obtained from a commercial provider (US Biomax, Inc.; cat. nos. HN802 and HN803c). Each TMA contained 80 individual cases including head and neck tumor tissue derived from oral, oropharyngeal and laryngeal SCC, as well as normal tissues. The tissue specimens were formalin-fixed and paraffin-embedded (FFPE). All TMA slides consisted of one single FFPE core per case (HNSCC and normal tissue).

A total of 11 non-SCC cases and 8 cases representing HNSCC metastases were excluded from the analysis. Additionally, 4 overlapping cases from both TMAs had to be considered. A total of 5 cases were excluded due to missing tissue cores. For 7 HNSCC cases, no grading information was available. The detailed clinicopathological sample characteristics are listed in Table I.

IHC. IHC was performed as previously described (25). For IHC staining of TMAs, a rabbit monoclonal antibody against hnRNP K [dilution 1:250, $60 \mathrm{~min}$ at room temperature (RT),
Biozol Diagnostica Vertrieb GmbH, cat. no. LS-C138027], a rabbit polyclonal antibody against p16INK4A (dilution 1:250, 60 min at RT, Cell Signaling Technology, Inc., cat. no. 4824) and the ZytoChem Plus AP Polymer Kit (Zytomed Systems $\mathrm{GmbH}$, cat. no. POLAP-100) were used according to the manufacturer's instructions. An experienced pathologist determined IHC scores separately for tumor cell nuclei and cytoplasm by assessing the staining intensity for hnRNP K and p16, respectively, using the following scoring method: Lack of immunoreactivity (0), weak immunoreactivity (1) and strong immunoreactivity (2).

Cell culture and transient transfection. Cal-27 cells were obtained from ATCC (CRL-2095 ${ }^{\mathrm{TM}}$, lot no. 62278082) and UPCI-SCC-154 were obtained from DSMZ (UPCI-SCC-154 ACC 669 lot no. 2) and were authenticated by STR analysis. The HPV status was determined using a PCR-based assay by the respective companies. Both cell lines were routinely tested to ensure the absence of mycoplasma contamination. Cal-27 originated from a 56-year old man suffering from SCC of the tongue and were cultivated in DMEM GlutaMAX (Gibco; Thermo Fisher Scientific, Inc.) supplemented with $10 \%$ FCS (Roche Diagnostics $\mathrm{GmbH}$ ) at $37^{\circ} \mathrm{C}$ and $5 \% \mathrm{CO}_{2}$. UPCI-SCC-154 cells were originally derived from an oral SCC of a 54-year-old man and were incubated in MEM (with Earle's salts) supplemented with 10\% FCS, 2 mM L-glutamine plus non-essential amino acids (Roche Diagnostics $\mathrm{GmbH}$ ). According to the provider, PCR analyses confirmed Cal-27 cells as derived from an HPV-negative and UPCI-SCC-154 cells as derived from an HPV-positive tumor. For the transfection experiments, we used Lipofectamine ${ }^{\mathrm{TM}} 2000$ as the transfection reagent (Invitrogen; Thermo Fisher Scientific, Inc.), Silencer ${ }^{\circledR}$ Select negative control siRNA \#1 and Silencer ${ }^{\circledR}$ Select hnRNP K (sequence: 3'-AUAAUCAUAGGUUUCAUC Gta; 5'-CGAUGAAACCUAUGAUUAUtt; both from Thermo Fisher Scientific, Inc.). At $48 \mathrm{~h}$ after transfection, cells were harvested and treated according to the respective experimental procedures.

Radiation exposure. For radiation experiments, HNSCC cells were exposed to $240 \mathrm{kV}$ X-rays using the MaxiShot system (YXLON International $\mathrm{GmbH}$ ) including a $3-\mathrm{mm}$ beryllium filter at a plateau dose rate of $1 \mathrm{~Gy} / \mathrm{min}$ at $13 \mathrm{~mA}$. Applied doses were monitored with a PTW Unidose dosimeter (PTW Freiburg $\mathrm{GmbH}$ ). During irradiation experiments, control cells were stored under equivalent external environmental conditions to ensure comparability.

Immunoblotting. For western blot analyses, the XCell Sure Lock ${ }^{\mathrm{TM}}$ Mini-Cell Electrophoresis System was used. Equalization of protein concentrations was performed using the BCA Protein Assay Kit (both from Thermo Fisher Scientific, Inc.). A rabbit monoclonal antibody was used for hnRNP K detection (dilution 1:1,000; Biozol Diagnostica Vertrieb GmbH, cat. no. LS-C138027) and HRP-conjugated anti-GAPDH served as the loading control (dilution 1:10,000; Cell Signaling Technology, Inc., cat. no. 3683S). Acquisition of digital images was carried out with the myECL ${ }^{\mathrm{TM}}$ Imager system (Thermo Fisher Scientific, Inc.). For densitometry greyscale intensity, the values of hnRNP K and GAPDH were 
Table I. Association of nuclear and cytoplasmic hnRNP K expression levels (IHC score) in HNSCC tissues with the clinicopathological characteristics of 117 patients with HNSCC.

\begin{tabular}{|c|c|c|c|c|c|}
\hline \multirow[b]{2}{*}{ Characteristics } & \multirow[b]{2}{*}{ No. } & \multicolumn{4}{|c|}{ IHC score hnRNP K } \\
\hline & & Nuclear $^{\mathrm{a}}$ & P-value ${ }^{b}$ & Cytoplasmic $^{\mathrm{a}}$ & P-value ${ }^{b}$ \\
\hline Normal oral tissue & 15 & $0.60 \pm 0.23$ & 0.028 & $0.27 \pm 0.12$ & 0.3 \\
\hline HNSCC & 117 & $1.16 \pm 0.08$ & & $0.49 \pm 0.06$ & \\
\hline Sex & & & $<0.001$ & & 0.009 \\
\hline Male & 59 & $1.46 \pm 0.10$ & & $0.68 \pm 0.10$ & \\
\hline Female & 58 & $0.86 \pm 0.10$ & & $0.29 \pm 0.07$ & \\
\hline HPV status & & & 0.298 & & 0.685 \\
\hline p16-negative & 95 & $1.13 \pm 0.08$ & & $0.47 \pm 0.07$ & \\
\hline p16-positive & 22 & $1.32 \pm 0.18$ & & $0.55 \pm 0.16$ & \\
\hline Age, years & & & 0.791 & & 0.32 \\
\hline$<57$ & 58 & $1.14 \pm 0.11$ & & $0.43 \pm 0.09$ & \\
\hline$\geq 57$ & 59 & $1.19 \pm 0.10$ & & $0.54 \pm 0.09$ & \\
\hline UICC stage & & & 0.052 & & 0.003 \\
\hline I-III & 83 & $1.07 \pm 0.09$ & & $0.34 \pm 0.06$ & \\
\hline IV & 34 & $1.38 \pm 0.14$ & & $0.85 \pm 0.14$ & \\
\hline Grade $^{c}$ & & & 0.426 & & 0.545 \\
\hline G1 & 37 & $1.27 \pm 0.13$ & & $0.40 \pm 0.10$ & \\
\hline G2 & 49 & $1.04 \pm 0.12$ & & $0.59 \pm 0.11$ & \\
\hline G3 & 24 & $1.21 \pm 0.17$ & & $0.49 \pm 0.15$ & \\
\hline
\end{tabular}

${ }^{\mathrm{a}}$ Mean \pm SEM; ${ }^{\mathrm{b} K r u s k a l-W a l l i s ~ t e s t, ~ D u n n ' s ~ p o s t ~ h o c ~ t e s t ; ~}{ }^{\mathrm{c}}$ No grading information was available for 7 HNSCC cases. hnRNP K, heterogenous nuclear ribonucleoprotein K; HNSCC, head and neck squamous cell carcinoma; IHC, immunohistochemistry; UICC, Union for International Cancer Control.

determined using ImageJ software, v. 1.51 (National Institutes of Health) before referencing to the non-irradiated control values and calculating the hnRNP K/GAPDH ratios.

Immunofluorescence (IF) microscopy. IF microscopy was performed as previously described (26). For IF staining of HNSCC cells, TexasRed-conjugated Phalloidin (dilution 1:40, Invitrogen; Thermo Fisher Scientific, Inc.), mouse monoclonal anti-hnRNP K (dilution 1:1,000, Cell Signaling Technology, Inc.) and a rabbit polyclonal anti-p16INK4A (dilution 1:1,000, Cell Signaling Technology, Inc.) were used following $60 \mathrm{~min}$ of incubation at RT. The nuclei were stained using Fluoroshield Mounting Medium with DAPI (Abcam). Fluorescence labeling was carried out with Alexa Fluor ${ }^{\circledR}$ 488-conjugated goat polyclonal anti-mouse and Alexa Fluor ${ }^{\circledR}$ 488-conjugated goat polyclonal anti-rabbit antibodies (both from Thermo Fisher Scientific, Inc.; dilution 1:500). Image acquisition was performed with a Zeiss AxioImager 2i fluorescence microscope (Carl Zeiss AG) and the ISIS fluorescence imaging system (MetaSystems).

Apoptosis assay. Induction of apoptosis was examined $6 \mathrm{~h}$ post-IR using the Bio-Plex ${ }^{\circledR}$ Multiplex Immunoassay System and the Bio-Plex Pro ${ }^{\mathrm{TM}}$ RBM Apoptosis Panel 3 (both from Bio-Rad Laboratories, Inc.) according to the manufacturers' instructions.
Clonogenic survival assay. UPCI-SCC-154 and Cal-27 cells were seeded in 6-well plates (seeding densities for $0 \mathrm{~Gy}$ : 200 cells/well; $2 \mathrm{~Gy}$ : 600 cells/well) and cultured at $37^{\circ} \mathrm{C}$ for $24 \mathrm{~h}$. Subsequently, the cells were transfected or irradiated $(n=4)$. After 9 days, tumor cell colonies were fixed with $70 \%$ ethanol at RT for 10 min before staining with gentian violet at RT for $10 \mathrm{~min}$. Colonies containing $>50$ cells were manually counted using a Zeiss STEMI SV8 stereomicroscope (Carl Zeiss AG). Experiments were performed in quadruplicate.

CAM assay. The CAM tumor xenograft assay was performed as previously described $(27,28)$. Briefly, fertilized chicken eggs were incubated $\left(37^{\circ} \mathrm{C} ; 60 \%\right.$ relative air moisture), fenestrated and a silicone ring (diameter: $5 \mathrm{~mm}$ ) was placed on top of the vascularized CAM on day 7 of fertilization. HNSCC cells $\left(1.5 \times 10^{6}\right.$ cells/egg) were dissolved in a $1: 1$ solution of medium and Matrigel (BD Diagnostics) and grafted on the CAM surface within the ring. HNSCC xenografts were incubated for another 4 days, harvested, imaged and fixed in phosphate-buffered $4 \%$ formaldehyde solution at RT for $24 \mathrm{~h}$ (Roti-Histofix ${ }^{\circledR}$, Carl Roth $\mathrm{GmbH}$ ). After embedding in paraffin, the tumor blocks were serially cut at $5 \mu \mathrm{m}$ and the sections were mounted on slides coated with poly-L-lysine. For subsequent IHC staining, the following primary antibodies were used: Monoclonal rabbit anti-hnRNP K (dilution 1:250; Biozol Diagnostica Vertrieb GmbH, cat. no. LS-C138027), monoclonal mouse 
Table II. Association of hnRNP K IHC score with the analyzed categories (tissue, sex and stage) among 117 HNSCC cases and 15 normal oral tissue samples.

\begin{tabular}{|c|c|c|c|c|}
\hline \multirow{2}{*}{$\begin{array}{l}\text { Model } \\
\text { Nuclear hnRNP K IHC score }>0^{\mathrm{a}}\end{array}$} & \multirow[t]{2}{*}{ OR } & \multicolumn{2}{|c|}{$95 \%$ CI } & P-value \\
\hline & & & & 0.004 \\
\hline Normal tissue & 1 & & & \\
\hline HNSCC & 5.5 & 1.8 & 17.5 & \\
\hline Nuclear hnRNP K IHC score $2^{\mathrm{a}}$ & & & & $<0.001$ \\
\hline Female & 1 & & & \\
\hline Male & 4.9 & 2.2 & 10.9 & \\
\hline Cytoplasmic hnRNP K IHC score $>0^{a}$ & & & & 0.005 \\
\hline Female & 1 & & & \\
\hline Male & 3.2 & 1.5 & 7.2 & \\
\hline Cytoplasmic hnRNP K IHC score $2^{\mathrm{a}}$ & & & & 0.002 \\
\hline I-III stage ${ }^{b}$ & 1 & & & \\
\hline IV stage $\mathrm{b}^{\mathrm{b}}$ & 7.1 & 2.0 & 25.1 & \\
\hline
\end{tabular}

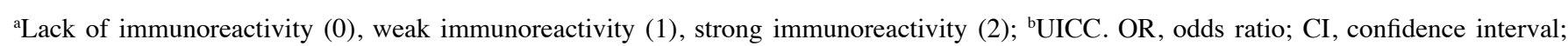
hnRNP K, heterogenous nuclear ribonucleoprotein K; HNSCC, head and neck squamous cell carcinoma; IHC, immunohistochemistry; UICC, Union for International Cancer Control.

anti-Ki-67 and monoclonal mouse anti-desmin (dilution 1:100; both from Dako; Agilent Technologies, Inc., cat. nos. M7240 and M0760).

Tumor volume $\left(\mathrm{TV} ; \mathrm{mm}^{3}\right)$ was calculated according to the formula: TV=length $(\mathrm{mm}) \mathrm{x}$ width $^{2}(\mathrm{~mm}) \mathrm{x} \pi / 6(29)$.

Statistical analysis. For calculation of differences between IHC expression levels, clonogenic survival and cleaved caspase-3 expression, Kruskal-Wallis test was performed followed by the appropriate post hoc test as indicated in the figure legends using GraphPad Prism v.5.00 (GraphPad Software, Inc.) or SigmaPlot 14.0 (Systat Software, Inc.). The plating efficiency (PE) and surviving fraction (SF) of colony formation assays were calculated as follows: $\mathrm{PE}=$ (colonies counted)/(cells seeded per well) $x 100 ; \mathrm{SF}=($ colonies counted $) /[$ (cells seeded per well $) \times(\mathrm{PE} / 100)]$. According to the linear-quadratic model of cell survival $\left(S=e^{-\alpha^{*} \mathrm{D}-\beta^{*} \mathrm{D}^{\wedge} 2}\right)$ we calculated the parameters $\alpha$ and $\beta$ by non-linear regression analysis using SigmaPlot 14.0 (Systat Software, Inc.) and considered $\mathrm{P}<0.05$ as statistically significant.

\section{Results}

Strong hnRNP K expression in HNSCC is associated with advanced tumor stage and male sex. Two sequential sections of tissue samples from 117 HNSCC patients and 15 samples of non-neoplastic oropharyngeal epithelium were immunostained for hnRNP K and p16 (Fig. 1). Nuclear, but not cytoplasmic, immunoreactivity with the antibody against hnRNP K was significantly elevated in HNSCC tumor samples vs. normal epithelium (Table I). Accordingly, the odds ratio for nuclear hnRNP K IHC scores $>0$ was found to be significantly increased in HNSCC tissue compared with normal oral tissue by 5.5 [95\% confidence interval (CI): 1.8-17.5; $\mathrm{P}=0.004$; Table II].

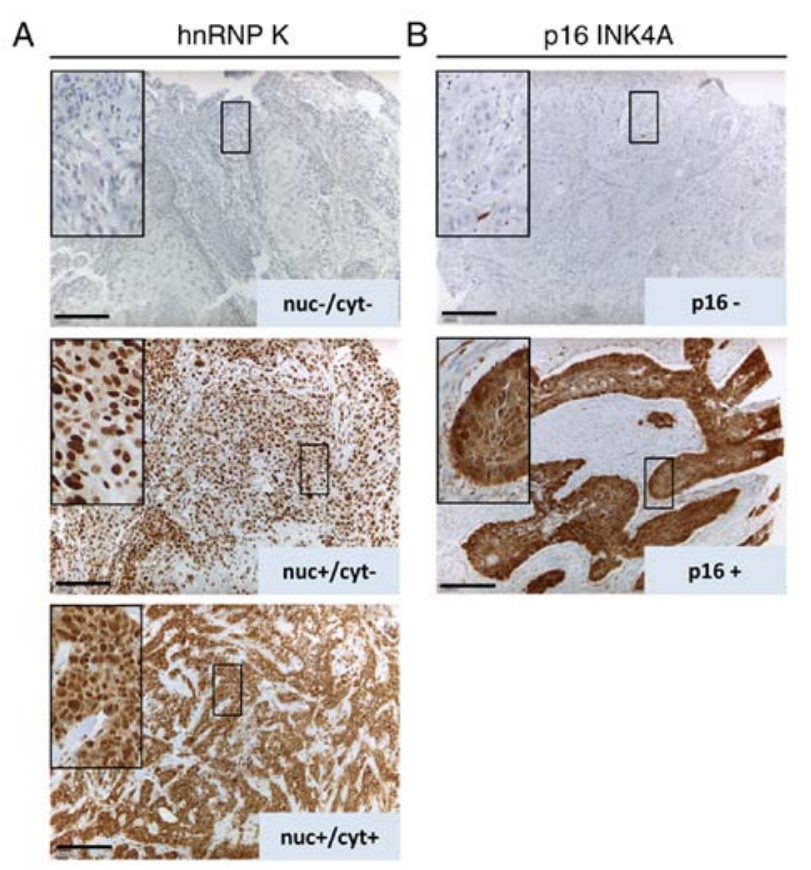

Figure 1. (A) Representative images of HNSCC tissue samples for hnRNP K IHC staining focusing on nuclear (nuc) and cytoplasmic (cyt) hnRNP K expression (scale bar, $200 \mu \mathrm{m}$ ): hnRNP K-negative (upper panel), nuclear hnRNP K expression (middle panel) and hnRNP K nuclear and cytoplasmic expression (lower panel). (B) Representative images of p16 INK4A-negative (upper panel) and -positive (lower panel) HNSCC samples. Staining for p16INK4A served as a surrogate marker for HPV-positive HNSCC. HNSCC, head and neck squamous cell carcinoma; hnRNP $\mathrm{K}$, heterogenous nuclear ribonucleoprotein $\mathrm{K}$; IHC, immunohistochemistry; HPV, human papillomavirus.

Furthermore, elevated cytoplasmic hnRNP K levels were observed in tissue samples from tumors with a high clinical stage [Union for International Cancer Control (UICC) IV, Table I]. Advanced tumor progression was shown to significantly increase the odds ratio by 7.1 (95\% CI: 2.0-25.1; 


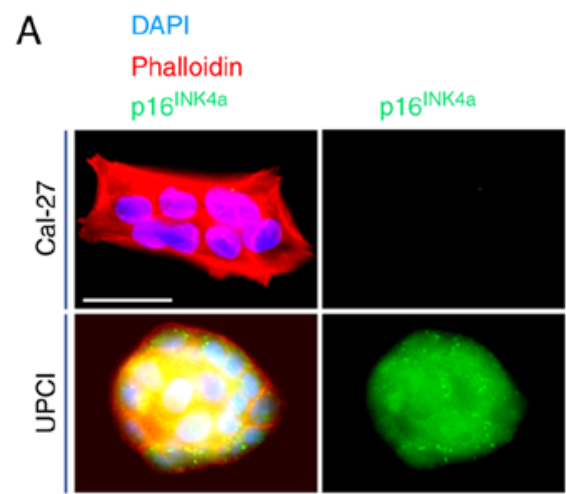

C

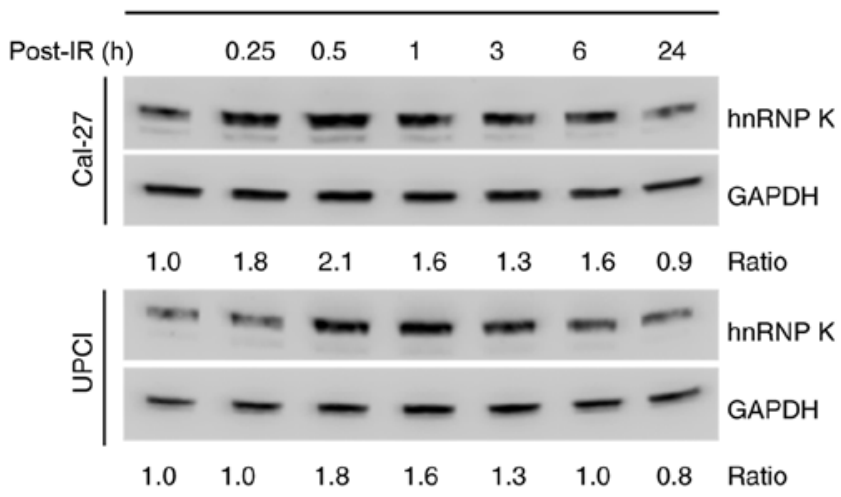

B

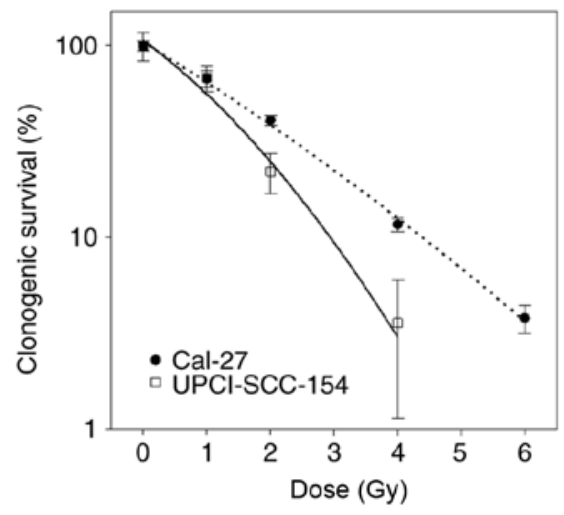

D

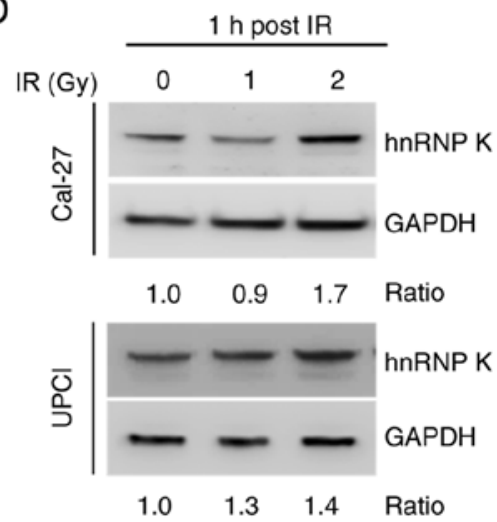

$\mathrm{E}$

\begin{tabular}{ll}
\multicolumn{1}{c}{ Cal-27 } & \multicolumn{1}{c}{ UPCI } \\
\cline { 3 - 3 } DAPI & DAPI \\
Phalloidin & Phalloidin
\end{tabular}

hnRNP K

hnRNP K

hnRNP K

hnRNPK
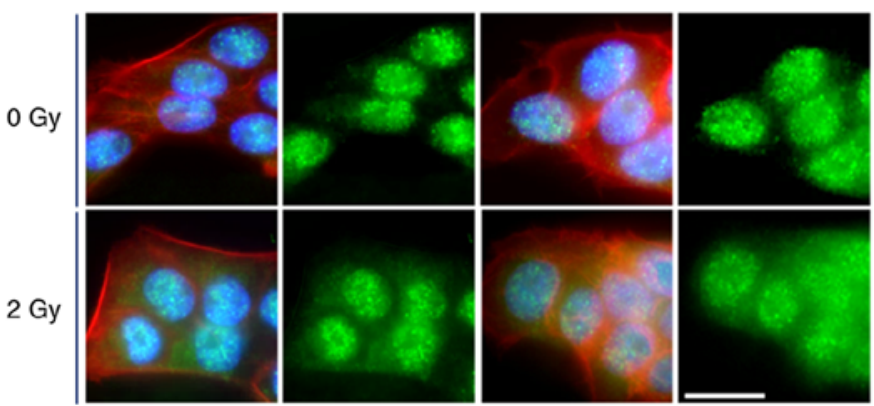

Figure 2. (A) Representative images of HNSCC cell clusters stained for p16INK4a using immunofluorescence illustrate the HPV status of Cal-27 (HPV-negative) and UPCI-SCC 154 (HPV-positive) cells (scale bar, $40 \mu \mathrm{m}$ ). (B) Clonogenic survival assay of the HNSCC cell lines Cal-27 and UPCI-SCC 154 after 9 days. Data are presented as mean \pm SD of 4 independent experiments. (C) Representative immunoblots demonstrate a rapid increase in cellular hnRNP K levels induced by IR ( 2 Gy), reaching maximum levels after 30-60 min before normalization of cellular hnRNP K levels within 24 h. Ratios represent hnRNP K/GAPDH referenced to non-irradiated control. (D) Dose-dependent accumulation of cellular hnRNP K $1 \mathrm{~h}$ after IR. (E) Immunofluorescence microscopy indicated cytoplasmic hnRNP K accumulation $1 \mathrm{~h}$ after irradiating cells with 2 Gy (scale bar, $20 \mu \mathrm{m}$ ). HNSCC, head and neck squamous cell carcinoma; hnRNP K, heterogenous nuclear ribonucleoprotein K; HPV, human papillomavirus; IR, ionizing radiation.

$\mathrm{P}=0.002$ ) for the presence of strong cytoplasmic hnRNP $\mathrm{K}$ immunoreactivity (IHC score 2) in the analyzed HNSCC samples (Table II).

Both strong nuclear and cytoplasmic hnRNP K immunoreactivity in HNSCC was shown to be statistically significantly associated with male sex (Table I). In line with these findings, the odds ratio for the presence of a higher hnRNP K expression was found to be significantly increased in male compared with female patients for both subcellular localizations, as shown in Table II.
By contrast, there was no significant difference between the mean values of hnRNP K IHC scores according to the p16 status as a surrogate marker of HPV-induced tumorigenesis or any other patient characteristics (Table I).

Accumulation of hnRNP $K$ in HNSCC cells as response to IR independent of HPV status. First, Cal-27 cells were verified as non-HPV- and UPCI-SCC-154 cells as HPV-associated HNSCC cell lines by p16INK4A immunostaining followed by fluorescence microscopy (Fig. 2A). Contrary to Cal-27, 
A
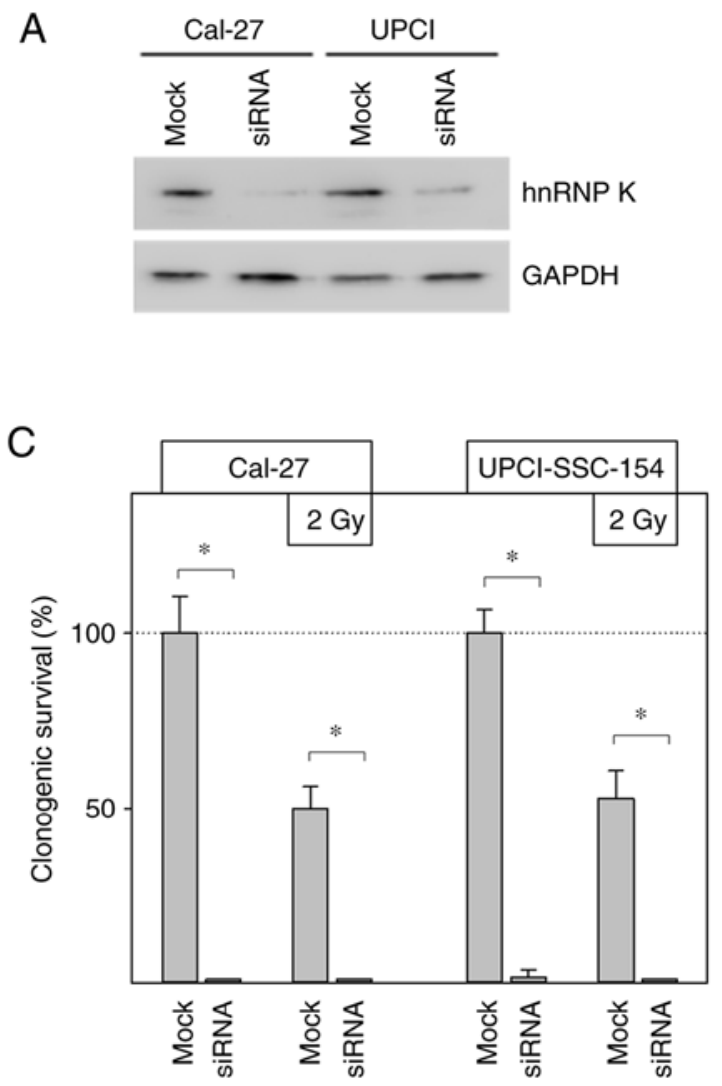

$\mathrm{B}$

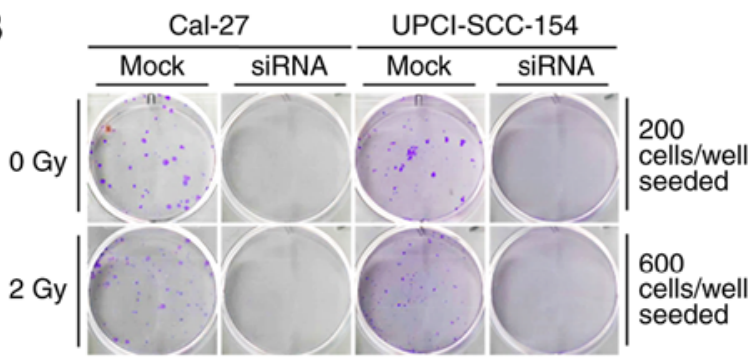

D



Figure 3. (A) Effective hnRNP K knockdown by transient transfection was verified by immunoblotting. Mock transfection served as control. (B) Representative images of colonies after 9 days of incubation. (C) Statistical analysis of clonogenic survival assays during hnRNP K knockdown. All experiments were carried out in quadruplicate. Data are presented as mean \pm SD. ${ }^{*} \mathrm{P}<0.05$ (Kruskal Wallis test, Tukey's post hoc test). (D) ELISA showed significantly increased levels of cellular active caspase-3 in Cal-27 and UPCI-SCC-154 cells parallel to hnRNP K knockdown. Data are presented as mean \pm SD. ${ }^{*}<<0.05$; n.s., not significant; $\mathrm{n}=6$ (Kruskal Wallis test, Holm-Sidak post hoc test). hnRNP K, heterogenous nuclear ribonucleoprotein K.

a dose of 6 Gy completely abrogated the clonogenicity of UPCI-SCC-154 cells (Fig. 2B). Based on the linear-quadratic model of cell survival, the linear $(\alpha)$ and quadratic $(\beta)$ constants of the fitted dose effect curves were determined (Fig. $2 \mathrm{~B} ; \mathrm{R}^{2}$ of the fit 0.997 for Cal-27 and 0.989 for UPCI-SCC-154) and calculated the $\alpha / \beta$-ratio in Gy. For UPCI-SCC-154 cells, a lower $\alpha / \beta$-ratio $(6.39 \mathrm{~Gy} ; 0.5164 / 0.0808)$ was found compared to Cal-27 cells (23.63 Gy; 0.4395/0.0186). These results revealed a higher radiosensitivity and a lower fractionation sensitivity for HPV-associated UPCI-SCC-154 cells compared to Cal-27 cells.

Acute radiation exposure using a clinically relevant individual dose of 2 Gy increased cellular hnRNP K concentrations in both cell lines, displaying a maximum plateau phase between 0.5 and $1 \mathrm{~h}$ post-exposure before reaching initial values after $24 \mathrm{~h}$, as shown by immunoblotting (Fig. 2C). This effect appeared to be dose-dependent when analyzing cells $1 \mathrm{~h}$ after radiation exposure (Fig. 2D).

To analyze radiation-induced changes in hnRNP K expression and subcellular localization, IF microscopy we additionally performed. Upon applying IR at $2 \mathrm{~Gy}$, both cell lines responded with cytoplasmic hnRNP K accumulation within $1 \mathrm{~h}$ (Fig. 2E).

Interestingly, all observed radiation-induced changes in the expression levels and cellular localization of hnRNP K were independent of the HPV status of the cell lines investigated.
Transient hnRNP K knockdown induces apoptosis in HNSCC cells. Knockdown experiments based on hnRNP K siRNA transfection were performed to specify the functional effects of cellular hnRNP K on the radiation response of HNSCC cells. The efficiency of the transient hnRNP K knockdown was verified by immunoblotting (Fig. 3A) and its persistence after 4 days was demonstrated by CAM experiments (Fig. 4A and B).

Clonogenic survival assays significantly impaired the clonogenic ability in both HNSCC cell lines deficient of endogenous hnRNP K (Fig. 3B and C). Cal-27 cells, in particular, completely lost their ability to form colonies under this condition. In UPCI-SCC-154 cells, complete abrogation of colony formation was achieved by combined treatment with hnRNP K knockdown and 2 Gy IR.

To highlight the potential role of apoptosis in this observation, the cellular levels of the pro-apoptotic protein active caspase-3 were analyzed (Fig. 3D). Radiation alone (2 Gy) significantly increased active caspase-3 by $\sim 1.3$-fold under control conditions (mock) in both investigated HNSCC cell lines. After additional hnRNP K knockdown, cellular active caspase-3 levels were found to be elevated even up to 3-fold. However, this finding reached statistical significance level for Cal-27 cells only.

In summary, these findings indicate enhanced apoptosis of HNSCC cells as the main cause for the deprivation of clonogenicity following loss of cellular hnRNP K, and confirmed 
A
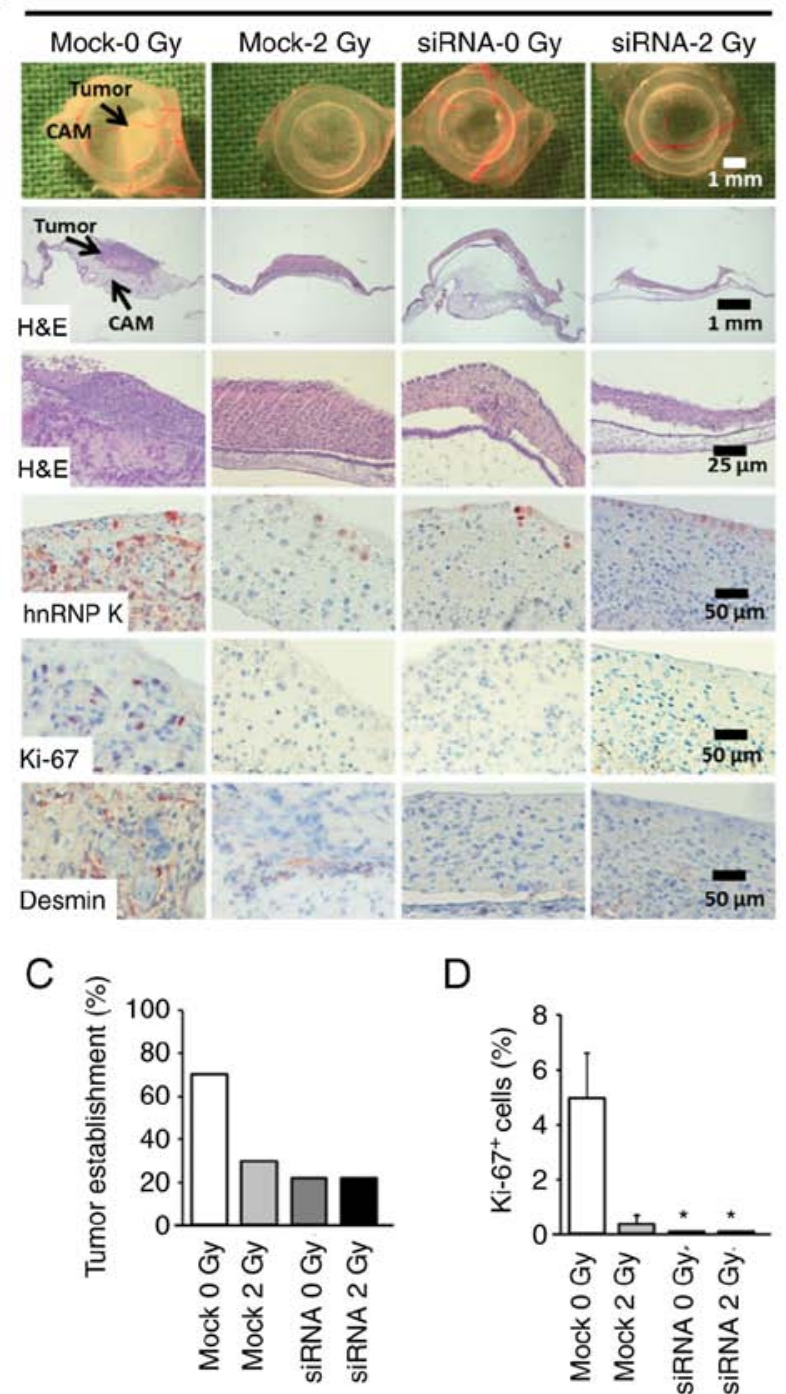

B

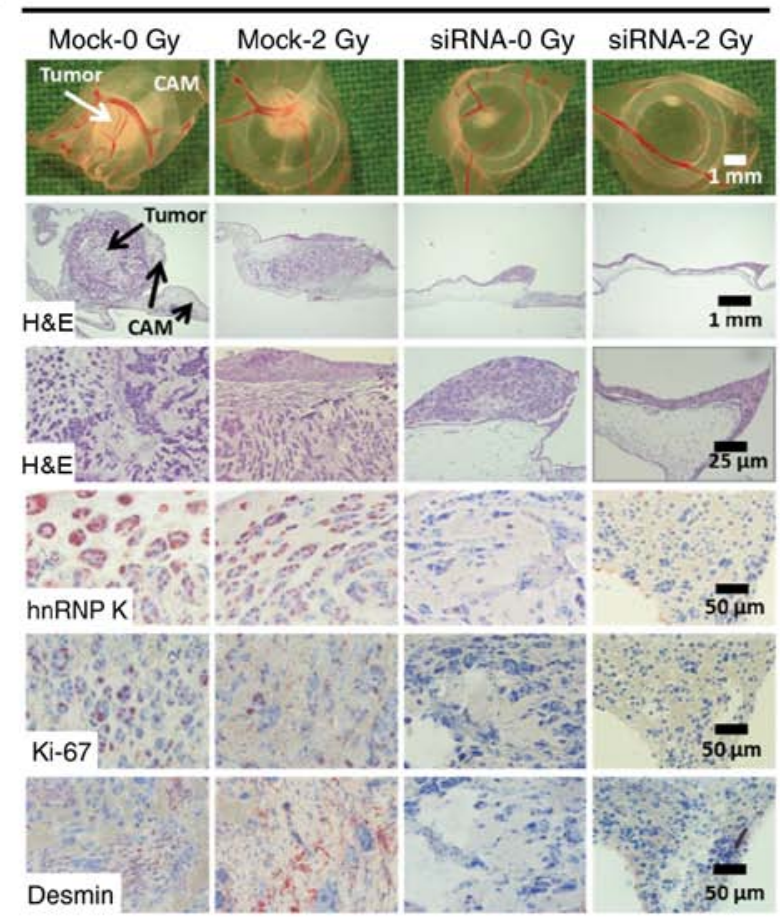

E

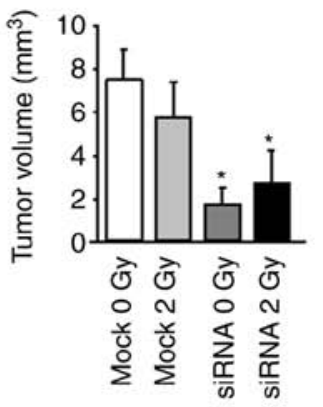

F



Figure 4. Knockdown of hnRNP K inhibits growth of HNSSC xenografts on the chick egg CAM in vivo. Cells (mock or siRNA, \pm irradiation) were seeded on the CAM of fertilized chick eggs 7 days after the start of incubation $\left(1.5 \times 10^{6}\right.$ cells/egg in medium/Matrigel 1:1). After an incubation period of 4 days at $37^{\circ} \mathrm{C}$, the tumors were collected, imaged, fixed and embedded in paraffin for immunohistochemical analysis. Sections (5 $\mu$ m) were stained for hnRNP $\mathrm{K}$, proliferation marker Ki-67 and the angiogenesis marker desmin. Each group contained 9-10 tumor-bearing eggs. (A and B) Representative images of tumor xenografts immediately after extraction (1st row), overview of tumor and underlying CAM tissue (H\&E staining, 2nd and 3rd rows), immunohistochemical staining of hnRNP K-expressing cells (4th row), Ki-67 ${ }^{+}$proliferative cells (5th row) and desmin ${ }^{+}$pericytes indicating angiogenesis (6th row). (C) Percentage of solid tumor formation of Cal-27 cells 4 days after xenotransplantation (9-10 tumors/group). (D) Percentage of proliferating Ki-67 cells in Cal-27 xenografts. A total of 261-358 cells from each tumor were evaluated. Data are presented as the mean \pm SEM of 4 tumors/group. (E) Mean tumor volume of UPCI-SCC-154 cancer xenografts 4 days after xenotransplantation as assessed immediately after extraction. Tumor volume was calculated according to the formula: $\pi / 6 \mathrm{x}$ length $\mathrm{x}$ width ${ }^{2}$. Mean of 9-10 tumors/group. (F) Percentage of proliferating Ki-67 ${ }^{+}$cells. A total of $298-632$ cells from each tumor were evaluated. Data are presented as the mean \pm SEM of 5 tumors/group. ${ }^{*} \mathrm{P}<0.05$ vs. control (mock 0 Gy) (Kruskal Wallis test, Dunnett's post hoc test). hnRNP K, heterogenous nuclear ribonucleoprotein K; HNSCC, head and neck squamous cell carcinoma; CAM, chorioallantoic membrane; H\&E, hematoxylin and eosin.

the involvement of hnRNP $\mathrm{K}$ in the radioresistance of HNSCC cells.

hnRNP K knockdown inhibits HNSCC xenograft formation on the CAM in vivo. The CAM model was used to investigate the impact of cellular hnRNP K levels on the tumor-forming capacity of HNSCC cells in vivo. HNSCC cells with hnRNP K knockdown and/or irradiation were seeded on the CAM of fertilized chicken eggs. The resulting tumors were imaged and harvested 4 days later. IHC of the FFPE tumor sections confirmed effective knockdown of hnRNP K (Fig. 4A and B). The tumor forming capacity of Cal-27 cells was markedly decreased by both hnRNP K knockdown and irradiation. In both conditions, macroscopic analysis of the tumor volumes was not possible due to poor tumor establishment in 30\% (mock 2 Gy) and 22\% (siRNA mock, siRNA 2 Gy) of the grafted eggs compared to $70 \%$ in the control group (mock) (Fig. 4C). This was confirmed by an almost complete lack of staining for the proliferation marker Ki-67 (Fig. 4D).

By contrast, UPCI-SCC-154 cells demonstrated a strong tumor forming capacity. Knockdown of hnRNP K resulted in significantly reduced tumor volume, impairment of proliferation, as indicated by Ki-67 immunostaining, and loss of tumor vascularization, as shown by desmin immunostaining, whereas irradiation alone showed minor efficacy (Fig. 4B, E and F). 


\section{Discussion}

The aim of the present study was to analyze the expression levels of hnRNP $\mathrm{K}$ in HNSCC, with an additional focus on its role in HNSCC radioresistance with respect to individual HPV status and sex. These investigations were based on the established role of hnRNP $\mathrm{K}$ in radioresistance of various malignant tumors (20), the higher radiosensitivity of HPV-associated HNSCC $(4,8)$ and sex-dependent prognosis of HNSCC patients $(30,31)$.

Significant higher nuclear, but not cytoplasmic, expression levels of hnRNP K were detected in HNSCC compared with normal oral mucosa samples, which was in line with previous findings $(16,19,32)$. This observation further supports the increasing evidence of high hnRNP K expression in cancerous/malignant tissues.

Furthermore,hnRNPKexpression was previously suggested as a prognostic biomarker associated with worse outcome (11). Consistently, the capability of hnRNP K to promote migration, angiogenesis and the expression of matrix metalloproteinases as prerequisites for invasion have previously been discussed as key contributing factors to a more malignant phenotype of hnRNP K-overexpressing tumors $(33,34)$. Furthermore, cytoplasmic hnRNP K levels were herein demonstrated to be significantly associated with advanced HNSCC tumor stages, which are characterized either by extensive infiltration by the primary tumor or by the presence of distant metastasis (UICC stage IV). In line with these observations, particularly aberrant cytoplasmic hnRNP K localization has been linked to unfavorable tumor characteristics and patient outcome, including patients with $\operatorname{HNSCC}(16,19)$.

IHC demonstrated a significantly stronger hnRNP K expression (both nuclear and cytoplasmic) in male compared to female patients. To the best of our knowledge, this is the first study to describe a sex-specific difference in hnRNP K expression in HNSCC tumor samples. A previous study also reported higher hnRNP K levels in HNSCC from male patients; however, the difference did not reach statistical significance (16). The finding of higher hnRNP K expression in male patients is of particular interest, since the number of studies analyzing the impact of sex on HNSCC biology and prognosis is limited and, in most studies, male patients are significantly over-represented in the investigated cohort $(16,30,35)$. Interestingly, two recent studies highlighted a significantly increased hazard ratio for male patients for HNSCC-related mortality $(30,31)$.

The mechanisms underlying this phenomenon remain to be fully elucidated; however, given the results presented herein, differences in hnRNP K expression may, at least in part, contribute to a worse prognosis in male HNSCC patients.

Despite our hypothesis, hnRNP K response to irradiation was not found to be dependent on HPV status. The higher baseline radiosensitivity of HPV-associated HNSCC cells was confirmed, while upregulation and cytoplasmic redistribution of hnRNP K upon exposure to IR were similar in both cell lines. siRNA-based knockdown of hnRNP K significantly increased the levels of active caspase-3 in HNSCC cells, indicating induction of apoptosis. Comparable initiation of programmed cell death due to hnRNP K knockdown has been previously demonstrated in various cancer cells $(17,18,36,37)$. However, loss of hnRNP K exerted no synergistic effect on the radiosensitivity of HNSCC cell lines, irrespective of their HPV status.

Further analyses using the CAM assay as an in vivo tumor xenograft model confirmed that knockdown of hnRNP K diminished the tumor-forming ability of HNSCC cells. Interestingly, a concomitant decrease in neovascularization and proliferation index within the tumor xenografts was observed upon hnRNP K knockdown. In Cal-27 cells, hnRNP K knockdown prevented tumor establishment. These observations may mainly be due to apoptosis induction, as demonstrated in vitro, combined with the abrogation of clonogenic survival upon hnRNP K knockdown in HNSCC cells. Furthermore, an essential role of hnRNP K in tumor-related angiogenesis has been described (33). At the molecular level, hnRNP K-dependent translation of vascular endothelial growth factor mRNA was demonstrated in renal tubular epithelial cells $(38,39)$. Thus, an impairment of tumor vascularization induced upon hnRNP K knockdown and a resulting lack of nutrients may explain the reduced proliferation index observed in HNSCC xenografts.

Taken together, the findings of the present study demonstrated that hnRNP K is overexpressed in HNSCC compared with normal squamous epithelium, and that high levels of hnRNP K are statistically associated with male sex and advanced tumor stage. There were no differences in hnRNP K expression levels between HPV- and non-HPV-associated HNSCC. IR led to quick upregulation and cytoplasmic redistribution of the hnRNP K protein, while hnRNP K knockdown enhanced IR-induced apoptosis and slowed down tumor growth in vitro and in vivo. This effect was independent of the HPV status of the cellular tumor model. Therefore, while hnRNP K appears to play a key role in tumorigenesis and evasion of apoptosis upon exposure to IR, the clinically observed radiosensitivity of HPV-associated HNSCC appears to be independent of hnRNP K expression levels in these tumors.

\section{Acknowledgements}

The authors would like to express their gratitude to Roland Ridi, Claudia Schlosser and Andreas Huebsch for their excellent technical assistance, to Barbara Couson and Jianqing Chen for performing the colony formation assays, and to Eva Winkler for supporting the grafting of the HNSCC cells.

\section{Funding}

No funding was received.

\section{Availability of data and materials}

The datasets generated and/or analyzed during the present study are available from the corresponding author on reasonable request.

\section{Authors' contributions}

Individual scientists contributed to the present work as follows: JK and SE: Conceptualization and methodology. SH and JK: CAM experiments and statistics. JK, SH, TP, CH, AR, MP and SE: Data analysis, writing, reviewing and editing of 
the manuscript. SK, $\mathrm{CH}$ and SE: IHC analysis and statistics. JK and SE: In vitro experimental investigation. SE: Project administration and supervision. All authors read and approved the final manuscript.

\section{Ethics approval and consent to participate}

Not applicable.

\section{Patient consent for publication}

Not applicable.

\section{Competing interests}

All the authors declare that they have no competing interests.

\section{References}

1. Torre LA, Bray F, Siegel RL, Ferlay J, Lortet-Tieulent J and Jemal A: Global cancer statistics, 2012. CA Cancer J Clin 65 : 87-108, 2015.

2. Bray F, Ferlay J, Soerjomataram I, Siegel RL, Torre LA and Jemal A: Global cancer statistics 2018: GLOBOCAN estimates of incidence and mortality worldwide for 36 cancers in 185 countries. CA Cancer J Clin 68: 394-424, 2018.

3. Franceschi S, Talamini R, Barra S, Baron AE, Negri E, Bidoli E, Serraino D and La Vecchia C: Smoking and drinking in relation to cancers of the oral cavity, pharynx, larynx, and esophagus in northern Italy. Cancer Res 50: 6502-6507, 1990.

4. Ang KK and Sturgis EM: Human papillomavirus as a marker of the natural history and response to therapy of head and neck squamous cell carcinoma. Semin Radiat Oncol 22: 128-142, 2012.

5. Chaturvedi AK: Epidemiology and clinical aspects of HPV in head and neck cancers. Head Neck Pathol 6 (Suppl 1): S16-S24, 2012.

6. Chaturvedi AK, Engels EA, Pfeiffer RM, Hernandez BY, Xiao W, Kim E, Jiang B, Goodman MT, Sibug-Saber M, Cozen W, et al Human papillomavirus and rising oropharyngeal cancer incidence in the United States. J Clin Oncol 29: 4294-4301, 2011.

7. Brouwer AF, Eisenberg MC and Meza R: Age effects and temporal trends in HPV-Related and HPV-Unrelated oral cancer in the United States: A multistage carcinogenesis modeling analysis. PLoS One 11: e0151098, 2016.

8. Fakhry C, Westra WH, Li S, Cmelak A, Ridge JA, Pinto H, Forastiere A and Gillison ML: Improved survival of patients with human papillomavirus-positive head and neck squamous cell carcinoma in a prospective clinical trial. J Natl Cancer Inst 100: 261-269, 2008

9. Liu C, Mann D, Sinha UK and Kokot NC: The molecular mechanisms of increased radiosensitivity of HPV-positive oropharyngeal squamous cell carcinoma (OPSCC): An extensive review. J Otolaryngol Head Neck Surg 47: 59, 2018.

10. Moody CA and Laimins LA: Human papillomavirus oncoproteins: Pathways to transformation. Nat Rev Cancer 10: 550-560, 2010.

11. Barboro P, Ferrari $\mathrm{N}$ and Balbi C: Emerging roles of heterogeneous nuclear ribonucleoprotein $\mathrm{K}$ (hnRNP K) in cancer progression. Cancer Lett 352: 152-159, 2014

12. Gallardo M, Hornbaker MJ, Zhang X, Hu P, Bueso-Ramos C and Post SM: Aberrant hnRNP K expression: All roads lead to cancer. Cell Cycle 15: 1552-1557, 2016.

13. Bomsztyk K, Denisenko O and Ostrowski J: hnRNP K: One protein multiple processes. Bioessays 26: 629-638, 2004.

14. Carpenter B, McKay M, Dundas SR, Lawrie LC, Telfer C and Murray GI: Heterogeneous nuclear ribonucleoprotein $\mathrm{K}$ is over expressed, aberrantly localised and is associated with poor prognosis in colorectal cancer. Br J Cancer 95: 921-927, 2006.

15. Chen LC, Chung IC, Hsueh C, Tsang NM, Chi LM, Liang Y, Chen CC, Wang LJ and Chang YS: The antiapoptotic protein, FLIP, is regulated by heterogeneous nuclear ribonucleoprotein $\mathrm{K}$ and correlates with poor overall survival of nasopharyngeal carcinoma patients. Cell Death Differ 17: 1463-1473, 2010.
16. Wu CS, Chang KP, Chen LC, Chen CC, Liang Y, Hseuh C and Chang YS: Heterogeneous ribonucleoprotein $\mathrm{K}$ and thymidine phosphorylase are independent prognostic and therapeutic markers for oral squamous cell carcinoma. Oral Oncol 48 : 516-522, 2012.

17. Chen X, Gu P, Xie R, Han J, Liu H, Wang B, Xie W, Xie W, Zhong G, Chen C, et al: Heterogeneous nuclear ribonucleoprotein $\mathrm{K}$ is associated with poor prognosis and regulates proliferation and apoptosis in bladder cancer. J Cell Mol Med 21: 1266-1279, 2017.

18. Eder S, Arndt A, Lamkowski A, Daskalaki W, Rump A, Priller M, Genze F, Wardelmann E, Port M and Steinestel K: Baseline MAPK signaling activity confers intrinsic radioresistance to KRAS-mutant colorectal carcinoma cells by rapid upregulation of heterogeneous nuclear ribonucleoprotein K (hnRNP K). Cancer Lett 385: 160-167, 2017.

19. Matta A, Tripathi SC, DeSouza LV, Grigull J, Kaur J, Chauhan SS, Srivastava A, Thakar A, Shukla NK, Duggal R, et al: Heterogeneous ribonucleoprotein $\mathrm{K}$ is a marker of oral leukoplakia and correlates with poor prognosis of squamous cell carcinoma. Int J Cancer 125: 1398-1406, 2009.

20. Moumen A, Masterson P, O'Connor MJ and Jackson SP: hnRNP K: An HDM2 target and transcriptional coactivator of p53 in response to DNA damage. Cell 123: 1065-1078, 2005.

21. Haley B, Paunesku T, Protic M and Woloschak GE: Response of heterogeneous ribonuclear proteins (hnRNP) to ionising radiation and their involvement in DNA damage repair. Int J Radiat Biol 85: 643-655, 2009.

22. Wiesmann N, Strozynski J, Beck C, Zimmermann N, Mendler S, Gieringer R, Schmidtmann I and Brieger J: Knockdown of hnRNPK leads to increased DNA damage after irradiation and reduces survival of tumor cells. Carcinogenesis 38: $321-328$, 2017.

23. Eder S, Lamkowski A, Priller M, Port M and Steinestel K: Radiosensitization and downregulation of heterogeneous nuclear ribonucleoprotein $\mathrm{K}$ (hnRNP K) upon inhibition of mitogen/extracellular signal-regulated kinase (MEK) in malignant melanoma cells. Oncotarget 6: 17178-17191, 2015.

24. Strozynski J, Heim J, Bunbanjerdsuk S, Wiesmann N, Zografidou L, Becker SK, Meierl AM, Gouveris H, Lüddens H, Grus F and Brieger J: Proteomic identification of the heterogeneous nuclear ribonucleoprotein $\mathrm{K}$ as irradiation responsive protein related to migration. J Proteomics 113: 154-161, 2015.

25. Steinestel K, Lennerz JK, Eder S, Kraft K and Arndt A: Invasion pattern and histologic features of tumor aggressiveness correlate with MMR protein expression, but are independent of activating KRAS and BRAF mutations in CRC. Virchows Arch 465: 155-163, 2014.

26. Liebau S, Steinestel J, Linta L, Kleger A, Storch A, Schoen M, Steinestel K, Proepper C, Bockmann J, Schmeisser MJ and Boeckers TM: An SK3 channel/nWASP/Abi-1 complex is involved in early neurogenesis. PLoS One 6: e18148, 2011.

27. Kuan SL, Fischer S, Hafner S, Wang T, Syrovets T, Liu W, Tokura Y, Ng DYW, Riegger A, Förtsch C, et al: Boosting antitumor drug efficacy with chemically engineered multidomain proteins. Adv Sci (Weinh) 5: 1701036, 2018.

28. Zuo Z, Syrovets T, Wu Y, Hafner S, Vernikouskaya I, Liu W, Ma G, Weil T, Simmet T and Rasche V: The CAM cancer xenograft as a model for initial evaluation of MR labelled compounds. Sci Rep 7: 46690, 2017.

29. Tomayko MM and Reynolds CP: Determination of subcutaneous tumor size in athymic (nude) mice. Cancer Chemother Pharmacol 24: 148-154, 1989.

30. Fakhry C, Westra WH, Wang SJ, van Zante A, Zhang Y, Rettig E, Yin LX, Ryan WR, Ha PK, Wentz A, et al: The prognostic role of sex, race, and human papillomavirus in oropharyngeal and nonoropharyngeal head and neck squamous cell cancer. Cancer 123: $1566-1575,2017$

31. Worsham MJ, Stephen JK, Chen KM, Mahan M, Schweitzer V, Havard S and Divine G: Improved survival with HPV among African Americans with oropharyngeal cancer. Clin Cancer Res 19: 2486-2492, 2013.

32. Roychoudhury P and Chaudhuri K: Evidence for heterogeneous nuclear ribonucleoprotein $\mathrm{K}$ overexpression in oral squamous cell carcinoma. Br J Cancer 97: 574-576, 2007.

33. Gao R, Yu Y, Inoue A, Widodo N, Kaul SC and Wadhwa R: Heterogeneous nuclear ribonucleoprotein K (hnRNP-K) promotes tumor metastasis by induction of genes involved in extracellular matrix, cell movement, and angiogenesis. J Biol Chem 288: 15046-15056, 2013 
34. Chung IC, Chen LC, Chung AK, Chao M, Huang HY, Hsueh C, Tsang NM, Chang KP, Liang Y, Li HP and Chang YS: Matrix metalloproteinase 12 is induced by heterogeneous nuclear ribonucleoprotein $\mathrm{K}$ and promotes migration and invasion in nasopharyngeal carcinoma. BMC Cancer 14: 348, 2014.

35. Ang KK, Zhang Q, Rosenthal DI, Nguyen-Tan PF, Sherman EJ, Weber RS, Galvin JM, Bonner JA, Harris J, El-Naggar AK, et al: Randomized phase III trial of concurrent accelerated radiation plus cisplatin with or without cetuximab for stage III to IV head and neck carcinoma: RTOG 0522. J Clin Oncol 32: 2940-2950, 2014.

36. Gao X, Feng J, He Y, Xu F, Fan X, Huang W, Xiong H, Liu Q, Liu W, Liu X, et al: hnRNPK inhibits GSK3 $\beta$ Ser9 phosphorylation, thereby stabilizing c-FLIP and contributes to TRAIL resistance in H1299 lung adenocarcinoma cells. Sci Rep 6: 22999, 2016.

37. Yang JH, Chiou YY, Fu SL, Shih IY, Weng TH, Lin WJ and Lin CH: Arginine methylation of hnRNPK negatively modulates apoptosis upon DNA damage through local regulation of phosphorylation. Nucleic Acids Res 42: 9908-9924, 2014.
38. Feliers D, Lee MJ, Ghosh-Choudhury G, Bomsztyk K and Kasinath BS: Heterogeneous nuclear ribonucleoprotein $\mathrm{K}$ contributes to angiotensin II stimulation of vascular endothelial growth factor mRNA translation. Am J Physiol Renal Physiol 293: F607-F615, 2007.

39. Sataranatarajan K, Lee MJ, Mariappan MM and Feliers D: PKCdelta regulates the stimulation of vascular endothelial factor mRNA translation by angiotensin II through hnRNP K. Cell Signal 20: 969-977, 2008.

(7) (9) This work is licensed under a Creative Commons Attribution-NonCommercial-NoDerivatives 4.0 International (CC BY-NC-ND 4.0) License. 